\title{
Bayesian Inference Approach to Inverse Problems in a Financial Mathematical Model
}

\author{
Yasushi Ota * \\ Okayama University of Science \\ Yu Jiang ${ }^{\dagger}$ \\ Shanghai University of Finance and Economics \\ Gen nakamura ${ }^{\ddagger}$ \\ Hokkaido University \\ Masaaki Uesaka $\S$ \\ Hokkaido University
}

February 28, 2019

\begin{abstract}
This paper investigates an inverse problem of option pricing in the extended Black-Scholes model. We identify the model coefficients from the measured data and attempt to find arbitrage opportunities in financial markets using a Bayesian inference approach. The posterior probability density function of the parameters is computed from the measured data. The statistics of the unknown parameters are estimated by Markov Chain Monte Carlo (MCMC), which explores the posterior state space. The efficient sampling strategy of MCMC enables us to solve inverse problems by the Bayesian inference technique. Our numerical results indicate that the Bayesian inference approach can simultaneously estimate the unknown drift and volatility coefficients from the measured data.
\end{abstract}

Keywords and Phrases: inverse problem; option pricing; Bayesian inference approach

\section{Introduction}

Financial derivatives are contracts that derive their value from the underlying asset, such as stocks, bonds, commodities, and exchange rates. The behavior of the

${ }^{*}$ This research was supported by KAKENHI (Grant number:18K03439). Faculty of Management, Okayama University of Science, 1-1 Ridaicyou, Okayama City, Okayama Japan 700-0005, E-mail:yota@mgt.ous.ac.jp

$\dagger$ Address: School of Mathematics Shanghai University of Finance and Economics 777 Guoding Rd., Shanghai,200433, P. R. China (E-mail:jiang.yu@mail.shufe.edu.cn)

${ }^{\ddagger}$ This research was supported by KAKENHI (Grant number:19K03554). Faculty of Science, Hokkaido University, nishi10 kitaku-kita20jyou Sapporo City, Hokkaido Japan 001-0020, E-mail:nakamuragenn@gmail.com

${ }^{\S}$ Faculty of Science, Hokkaido University, nishi10 kitaku-kita20jyou Sapporo City, Hokkaido Japan 001-0020, (E-mail:muesaka@es.hokudai.ac.jp)

*Email : yota@mgt.ous.ac.jp 
risk asset's price is modeled by a diffusion process $S_{t}$ satisfying

$$
d S_{t}=\mu\left(t, S_{t}\right) S_{t} d t+\sigma\left(t, S_{t}\right) S_{t} d W_{t},
$$

where $W_{t}$ denotes Brownian motion, and $\mu$ and $\sigma$ are often called the volatility and the drift(trend), respectively, of the risk asset's price. When $\mu$ and $\sigma$ are positive constants, $S_{t}$ is called the Black-Scholes model.

As is well known, under the assumption that no-arbitrage property of a financial market, the option price $u(t, S)$ is given by the following initial value problem

$$
\left\{\begin{array}{c}
\frac{\partial u}{\partial t}+\frac{1}{2} \sigma(t, S)^{2} S^{2} \frac{\partial^{2} u}{\partial S^{2}}+r S \frac{\partial u}{\partial S}-r u=0, \quad(t, S) \in[0, T) \times(0, \infty), \\
\left.u(t, S)\right|_{t=T}=\max (0, S-K),
\end{array}\right.
$$

where the interest rate $r$ is a nonnegative constant, and $K$ is the strike price and $T$ is the maturity of the underlying asset. The approach of the Black-Scholes model provides a useful, simple method of pricing inclusive of financial derivatives, risk premium, and default probability estimation. However, the theoretical prices of options with different strike prices which are calculated by the Black-Scholes equation differ from real market prices. In [6] and [18], taking this into account, we have extended Black-Scholes equation as follows :

$$
\left\{\begin{array}{cc}
\frac{\partial u}{\partial t}+\frac{1}{2} \sigma(t, S)^{2} S^{2} \frac{\partial^{2} u}{\partial S^{2}}+\mu(t, S) S \frac{\partial u}{\partial S}-r u=0, & (t, S) \in[0, T) \times(0, \infty), \\
\left.u(t, S)\right|_{t=T}=\max (0, S-K), & S \in(0, \infty) .
\end{array}\right.
$$

In different real markets handling the same risk and asset, an arbitrage opportunity often appears in the error $\mu-r$. Practitioners may also request a convenience yield $\mu-r$ from commodity markets. Suppose that $N$ arbitrage markets handle the same asset with price $s_{i}(i=1,2, \cdots, N)$ at a given time $t^{*}$, we try to identify $\mu\left(S_{i}\right)-r$ from the measured call option prices $u^{*}\left(S_{i}\right)$, at the same time $t^{*}$.

In this paper, our inverse problem seek the value of $\mu(S)-r$ from given

$$
\left.u(S, t)\right|_{t=t^{*}}=u^{*}(S), \quad S \in \omega,
$$

where $\omega$ is the interval and $u(t, S)$ satisfies (1.2).

Inverse option problems (IOP) in mathematical finance were pioneered by Dupire [5]. Assuming no arbitrage, he derived the option premium $U(T, K)$ as a solution $u(\cdot, \cdot ; T, K)$ to the dual equation of (1.2) with respect to the strike price $K$ and maturity $\mathrm{T}$ as follows :

$$
\frac{\partial U}{\partial T}-\frac{1}{2} \sigma(T, K)^{2} K^{2} \frac{\partial^{2} U}{\partial K^{2}}+r K \frac{\partial U}{\partial K}=0
$$


If the option price and its derivative can be determined for all possible $T$ and $K$, then the local volatility function $\sigma(T, K)$ can be directly derived from Eq.(1.3) as

$$
\sigma(T, K)^{2}=\frac{\frac{\partial U}{\partial T}+r K \frac{\partial U}{\partial K}}{\frac{1}{2} K^{2} \frac{\partial^{2} U}{\partial K^{2}}} .
$$

Using this approach, we can deduce the local volatility function from the quoted option prices in the financial market. However, this strategy is impractical because we cannot get numerically stable data. In fact, since the data in financial markets are usually scarce and noisy, we cannot guarantee that the right-hand side of (1.4) remains constantly positive. Moreover the second derivatives of the option prices for all possible $T$ and $K$ are difficult to obtain, and their inverse problems are generally ill-posed. Using a linearization method, Bouchouev and Isakov [2], Bouchouev et al. [3], and Ota and Kaji [19] considered the following form of the time-independent local volatility function $\sigma^{2}(K)$ :

$$
\frac{1}{2} \sigma^{2}(K)=\frac{1}{2} \sigma_{0}^{2}+f(K)
$$

where $f$ is a small perturbation of the constant $\sigma_{0}$. They transformed (IOP) to a Fredholm-type integral equation for $f(K)$ and numerically determined the timeindependent local volatility function. On the other hand, Mitsuhiro and Ota [18], Korolev et al. [12] and Doi and Ota [6] used the extended Black-Scholes equation (1.2) and then reconstructed the trend function by linearization method. The above studies provided point estimates of unknown parameters by exact determination or least squares optimization, without rigorously examining and considering the measurement errors in the inverse solutions. However, since most of the data in real financial markets are contaminated by measurement errors, uncertainties are ubiquitous. Further errors are introduced by linearizing the inverse problems or the inverse methodology; for example, discretizing the integral equation obtained by the linearization method.

In this paper, we reconstruct the parameters not by linearizing the inverse problems but by applying Bayesian inference to our inverse problem. Bayesian inference approach solves an inverse problem by formulating a complete probabilistic description of the unknowns and uncertainties from the given measured data (see [11]). Incorporating the likelihood function with a prior distribution, the Bayesian inference method provides the posterior probability density function (PPDF). Owing to the recent developments in Bayesian inference work, including Bayesian inference approach by efficient sampling methods such as Markov Chain Monte Carlo (MCMC), we can apply the Bayesian inference technique to inverse problems in remote sensing [8], seismic inversion [16], heat conduction problems [21], [22] and various other real-world problems. 
Dupire's method [5] requires the solution at all points or the second order gradient of the solution. On the other hand, MCMC method can avoid having this. Rather than applying a Dupire-type formula or the linearization method, we attempt a parameter reconstruction by a statistical method that simultaneously estimates the unknown trend and volatility coefficients from the measured data. In other words, we seek arbitrage opportunities by simultaneously estimating the gap in the given interest rate and the volatility. If our results reveal the existence of an arbitrage, then a profitable trading strategy in real financial markets is expected.

This paper is divided into five parts. Our inverse problem is mathematically formulated in Section 2. Section 3 outlines the general Bayesian framework for solving inverse problems and discusses the numerical exploration of the posterior state space by the MCMC method. In Section 4, we discretize our inverse problem and reconstruct the parameters by a numerical algorithm. We then discuss various aspects of our results through numerical examples. Concluding remarks are given in Section 5.

\section{Inverse option problems}

This section formulates an inverse problem which is defined in Introduction. In the following problem, the local volatility $\sigma(t, S)$ is a positive constant $\sigma_{0}>0$ and the trend $\mu(t, S)$ is a time-independent function in (1.2) under the suitable condition:

$$
\left.u(t, S)\right|_{t=T}=\max \{S-K, 0\},
$$

where $K$ is the stock price at the maturity date $T$. Upon the following change of variables and substitutions,

$$
y=\log \frac{S}{K}, \quad \tau=T-t
$$

then, the equation and initial condition of (1.2) in terms of

$$
\mu(y)=\mu\left(D e^{y}\right), \quad U(\tau, y)=u\left(T-\tau, K e^{y}\right) / D
$$

becomes

$$
\left\{\begin{array}{cc}
\frac{\partial U}{\partial \tau}=\frac{1}{2} \sigma_{0}^{2} \frac{\partial^{2} U}{\partial y^{2}}-\left(\frac{1}{2} \sigma_{0}^{2}-\mu(y)\right) \frac{\partial U}{\partial y}-r U, & (\tau, y) \in\left(0, \tau^{*}\right) \times \mathbf{R}, \\
\left.U(\tau, y)\right|_{\tau=0}=\max \left\{e^{y}-1,0\right\}, & y \in \mathbf{R}, \\
U\left(\tau^{*}, y\right)=U^{*}(y), & y \in \omega \subseteq \mathbf{R},
\end{array}\right.
$$

where $\tau^{*}=T-t^{*}>0, t^{*}$ is the current time and $\omega$ is an interval of $\mathbf{R}$. Equation (2.4) has unique solution $U$ in a suitable functional space (see Friedman, [7]). In 
our IOP, Eqs. (2.4) and (2.5) seek the values of $\mu(y)=r+f(y)$ and $\sigma_{0}$ from the given $U^{*}(y)$, where $f$ is a small perturbation of the interest rate $r$.

However, due to the nonlinearity of this inverse problem, the uniqueness and existence of its solution are hard to prove. The present paper attempts to reconstruct the parameters by a statistical method simultaneously estimates $\mu(y)=$ $r+f(y)$ and $\sigma_{0}$ from the measured data $U^{*}(y)$. In other words, we seek arbitrage opportunities by simultaneously estimating the gap from the given interest rate and the volatility.

Let us define $m$-dimensional vectors $Y, F(\theta)$ and $\varepsilon$ as follows :

$$
\begin{aligned}
\{Y\}_{j} & =U^{*}\left(y_{j}\right)=U\left(\tau^{*}, y_{j} ; \bar{\theta}\right)\left(1+\varepsilon_{j}\right) \\
\{F(\theta)\}_{j} & =U\left(\tau^{*}, y_{j} ; \theta\right) \\
\{\varepsilon\}_{j} & =\varepsilon_{j}
\end{aligned}
$$

where $y_{j}(j=1, \cdots, m)$ are the measurement points at $\tau^{*}, U\left(\tau^{*}, y_{j} ; \theta\right)$ solves the Cauchy problem (2.4) for the unknown parameters $\theta$ and $\varepsilon_{j}$ is the uncertainty (noise) in the market, assumed as white Gaussian noise. We then seek the parameters $\bar{\theta}$, which assumedly represent the true value of $\theta$, such that

$$
Y \simeq F(\theta)+\varepsilon
$$

\section{Bayesian inference approach to the IOP}

Recently, the Bayesian inference approach has been greatly extended through the development of analytical techniques such as MCMC (see [11]).

The Bayesian inference approach considers the parameters not as single valued, but as a probability distribution. The above-mentioned PPDF defines the parameter probability distribution estimated from the measured data. Mathematically, the PPDF is expressed as $f(\theta \mid Y)$ and is the product of the likelihood function $f(Y \mid \theta)$ and the prior density function $f(\theta)$. The underlying concept of Bayesian inference is Bayes' theorem, which relates the parameters $\theta$ and the observed data $Y$ as follows:

$$
f(\theta \mid Y)=\frac{f(Y \mid \theta) f(\theta)}{f(Y)} .
$$

Equation (3.1) states that given some observations $Y$, the posterior probability of a hypothesis is proportional to the product of its likelihood and its prior probability.

The present paper assumes that the random errors in Eqs. (2.5) are white Gaussian noise with a known standard deviation $\Sigma_{\varepsilon}$. The likelihood function $f(Y \mid \theta)$ is then given as

$$
f(Y \mid \theta)=\exp \left\{-\frac{(Y-F(\theta))^{T}(Y-F(\theta))}{2 \Sigma_{\varepsilon}^{2}}\right\} .
$$


The prior density function is simply assumed as $f(\theta)=U_{\left[-\theta_{0}, \theta\right]}$, where $\theta_{0}$ is a sufficiently large positive constant. The PPDF of the parameters $\theta$ can be written as follows:

$$
f(\theta \mid Y) \propto \exp \left\{-\frac{(Y-F(\theta))^{T}(Y-F(\theta))}{2 \Sigma_{\varepsilon}^{2}}\right\} .
$$

Here, the standard derivation $\Sigma_{\varepsilon}$ is known and can be regarded as a regularization parameter.

\subsection{MCMC methods}

In Bayesian inference, the complicated and intractable probabilistic models can be estimated by numerical sampling methods such as MCMC, which has been widely applied in recent years. The details of MCMC methods are given in Robert and Casella [20].

Monte Carlo simulation generates pseudo-random numbers for exploring posterior distributions. In the MCMC algorithm, the pseudo-random number is a Markov chain. The MCMC algorithm exploits the property of a Markov chain to generate pseudo-random numbers from a posterior distribution, even for a complicated model. It first constructs an ergodic Markov chain with a stationary distribution equaling the target distribution. By iterating the Markov chain transitions from suitable initial value, it eventually obtains the target distribution.

This paper employs a typical MCMC algorithm called the Metropolis-Hastings (M-H) algorithm (see Metropolis et al. [17]; Hastings [9]). The M-H (Algorithm 1) given below builds its Markov chain by accepting or rejecting samples extracted from a proposed distribution. This algorithm is generally used in Bayesian inference and is a powerful tool for solving inverse problems (cf. [11]).

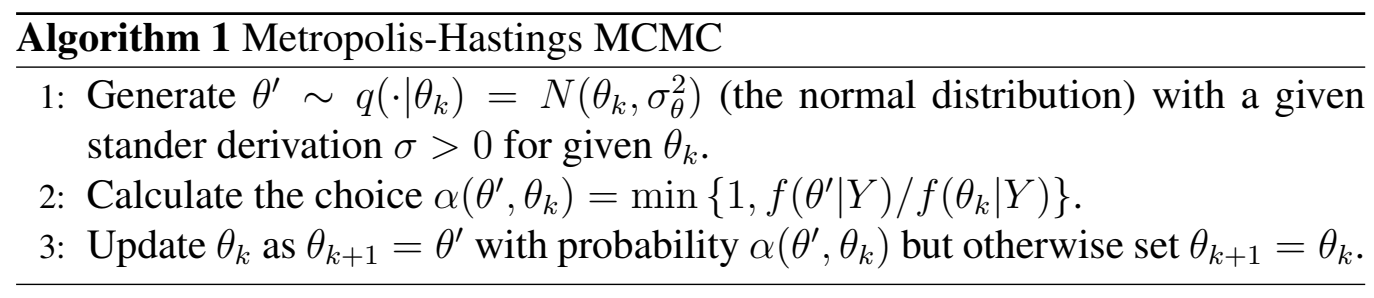

By running the $\mathrm{M}-\mathrm{H}$ algorithm, we can sample the distribution $f(\theta \mid Y)$, and usually the mean value of $\theta_{k}$, after a given burn-in time $k^{*}$. Unlike common Newton-type iterative regularization methods (for example, the Levenberg-Marquardt algorithm), the MCMC algorithm does not highly depend on the initial guess and always reaches the global minimum after a sufficiently long sampling time. 
Bayesian inference approach to inverse problems

\section{Numerical examples}

In this section, we generate numerically an exact artificial data set $F(\theta)$ and let (4.2) be the numerical data, where random error $\varepsilon$ contains both the random measurement error and the numerical error.

In the rest of this paper, we assume the trend $\mu(y)$ has the form :

$$
\mu(y)=r+\mu_{1} y
$$

where $\mu_{1}$ is the unknown constant. We also assume the measurement data $Y$ has the form :

$$
Y=F(\theta)+\varepsilon,
$$

where random error $\varepsilon$ contains both the random measurement error and the numerical error. By reconstructing the parameters by the $\mathrm{M}-\mathrm{H}$ method, we simultaneously estimate $\mu_{1}$ and $\sigma_{0}$ from the measured data $Y$ in Eqs.(4.2).

\subsection{Direct problems}

In this section, we assume $r=0$ and solve the direct problem for (2.4) by the numerical Crank-Nicholson scheme :

$$
\begin{aligned}
a_{j} U_{i+1, j+1}+(1+b) & U_{i+1, j}+c_{j} U_{i+1, j-1} \\
= & -a_{j} U_{i, j+1}+(1-b) U_{i, j}-c_{j} U_{i, j-1}
\end{aligned}
$$

where $U_{i, j}=U\left(t_{i}, y_{j}\right)$, and

$$
\begin{gathered}
a_{j}=-\frac{\Delta \tau}{4(\Delta y)^{2}}\left\{\sigma_{0}+\Delta y\left(-\frac{1}{2} \sigma_{0}+\mu_{1} y_{j}\right)\right\}, \\
b=\frac{\Delta \tau}{2(\Delta y)^{2}}, \\
c_{j}=-\frac{\Delta \tau}{4(\Delta y)^{2}}\left\{\sigma_{0}-\Delta y\left(-\frac{1}{2} \sigma_{0}+\mu_{1} y_{j}\right)\right\} .
\end{gathered}
$$

Here, we took a uniform grid

$$
\begin{aligned}
\tilde{\omega}=\left\{\left(\tau_{i}, y_{j}\right): \tau_{i} \in\left(0, \tau^{*}\right),\right. & y_{j} \in I_{15}=(-15,15) \\
& i=1,2, \cdots, M, j=1,2, \cdots, 200\}
\end{aligned}
$$

with artificial zero Dirichlet boundary conditions at $y=-15$ and 15 , and $\Delta \tau=$ $\tau_{i+1}-\tau_{i}=0.001, \Delta y=y_{j+1}-y_{j}=\frac{30}{199}$. 
The direct problem (2.4) can be given in the matrix form :

$$
\mathbf{u}_{i+1}=\mathbf{A}^{-1} \mathbf{B u}_{i}
$$

where $\mathbf{u}_{\mathbf{i}}=\left(U_{i, 2}, U_{i, 3}, \cdots, U_{i, 199}\right)^{T}, \mathbf{e}_{\mathbf{1 9 8}}=(0,0, \cdots, 0,1)^{T}$ and

$$
\begin{aligned}
& \mathbf{A}=\left(\begin{array}{cccccc}
1+b & a_{2} & 0 & 0 & \ldots & 0 \\
c_{3} & 1+b & a_{3} & 0 & \ldots & 0 \\
0 & c_{4} & 1+b & a_{4} & \ldots & 0 \\
\vdots & & \ddots & \ddots & \ddots & \vdots \\
0 & & & c_{198} & 1+b & a_{198} \\
0 & \ldots & & 0 & c_{199} & 1+b
\end{array}\right) \\
& \mathbf{B}=\left(\begin{array}{cccccc}
1-\tilde{b} & -a_{2} & 0 & 0 & \ldots & 0 \\
-c_{3} & 1-\tilde{b} & -a_{3} & 0 & \ldots & 0 \\
0 & c_{4} & 1-\tilde{b} & -a_{4} & \ldots & 0 \\
\vdots & & \ddots & \ddots & \ddots & \vdots \\
0 & & & -c_{198} & 1-\tilde{b} & -a_{198} \\
0 & \ldots & & 0 & -c_{199} & 1-\tilde{b}
\end{array}\right),
\end{aligned}
$$

where $\tilde{b}=b+\Delta \tau r$.

\subsection{Inverse problem solution by MCMC}

Table 1 shows the true values and parameter settings in Algorithm 1.

\section{Table 1: Parameter setting in Algorithm 1.}

\begin{tabular}{c||c|c||c|c}
\hline \hline True value & $\mu_{1}$ & 1 & $\sigma_{0}$ & 1 \\
\hline Others & $\Sigma_{\varepsilon}$ & $10^{-3}$ & $\sigma_{\theta}$ & $(0.01,0.01)$ \\
\hline \hline
\end{tabular}

In the following examples, the relative noise in all the observations $Y$ is assumed as $5 \%$ and the prior distribution $f(\theta)$ of unknowns is $\left(\mu_{1}, \sigma_{0}\right)=1$. That is, we can say $f_{\text {prior }}(\theta)=\mathbf{1}_{\left[\mu_{1}^{\min }, \mu_{1}^{\max }\right]}\left(\mu_{1}\right) \cdot \mathbf{1}_{\left[\sigma_{0}^{\min }, \sigma_{0}^{\max }\right]}\left(\sigma_{0}\right)$ and the intervals $\left[\mu_{1}^{\min }, \mu_{1}^{\max }\right]$ and $\left[\sigma_{0}^{\min }, \sigma_{0}^{\max }\right]$ are large enough so that all $\left(\mu_{1}, \sigma_{0}\right)$ 's appearing in the Markov chain fall into these intervals. Here, we set the the indicator function as

$$
\mathbf{1}_{A}(a)= \begin{cases}1 & a \in A \\ 0 & a \notin A\end{cases}
$$


Bayesian inference approach to inverse problems

General uniform distributions can be used for $f(\theta)$ if we use the prior-reversible proposal that satisfies $f(\theta) q\left(\theta^{\prime} \mid \theta\right)=f\left(\theta^{\prime}\right) q\left(\theta \mid \theta^{\prime}\right)$ (see for example [10]). On the other hand, if we choose $f(\theta)$ as a Gaussian distribution, this will turn out to be the Tikhonov regularization term in the cost function.

For comparison, we particularly consider the Levenberg-Marquardt algorithm $[13,15]$. That is, the recovery of $\theta=\left(\mu_{1}, \sigma_{0}\right)^{T}$ is computed by the iteration given by

$$
\theta_{k+1}=\theta_{k}+\left[F^{\prime}\left(\theta_{k}\right)^{T} F^{\prime}\left(\theta_{k}\right)+\lambda I\right]^{-1} F^{\prime}\left(\theta_{k}\right)^{T}\left(U-F\left(\theta_{k}\right)\right)
$$

where $F^{\prime}(a)$ is the Jacobian matrix and the parameter $\lambda$ is nonnegative. This algorithm can be implemented by an inner embedded program Isqcurvefit in MATLAB (B) $2018 a$.

\section{Example 1:}

The MCMC sampled $\left(\mu_{1}, \sigma_{0}\right)$ is shown in Figures 1 and 2, respectively. The recovered result is given in Table 2 showing a good recovery. For comparison, the converged recovery of $\left(\mu_{1}, \sigma_{0},\right)$ obtained by the Levenberg-Marquardt algorithm is also provided in Table 2.

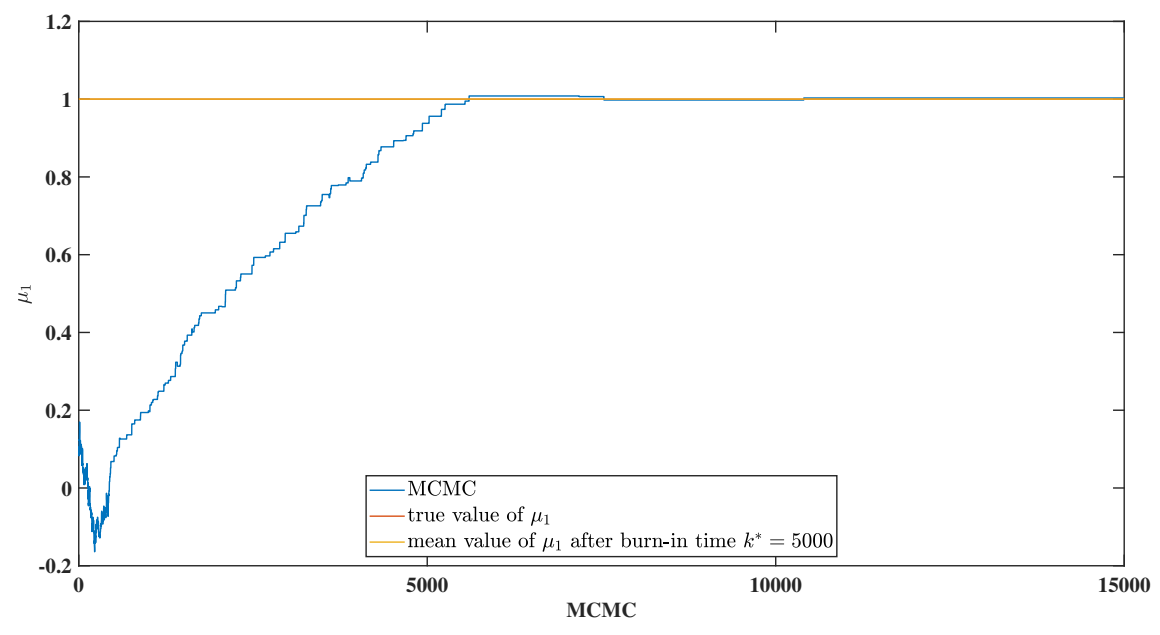

Figure 1: MCMC sampling of the parameter $\mu_{1}$. 


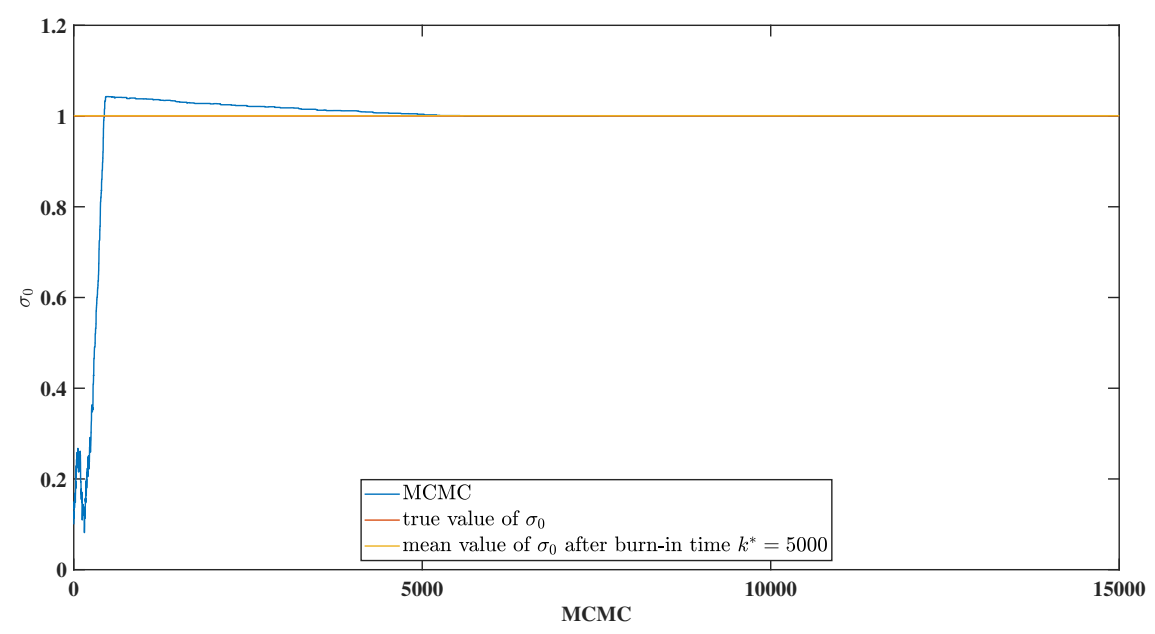

Figure 2: MCMC sampling of the parameters $\sigma_{0}$

Table 2: Recovery results of $\left(\mu_{1}, \sigma_{0}\right)$.

\begin{tabular}{c||c|c}
\hline \hline & $\mu_{1}$ & $\sigma_{0}$ \\
\hline \hline Initial guess & 0.1 & 0.1 \\
\hline Mean value after burn-in time $k^{*}=5000$ & 1.0008 & 1.0002 \\
\hline Result of Levenberg-Marquardt algorithm & 1.0020 & 1.0001 \\
\hline True value & 1 & 1 \\
\hline \hline
\end{tabular}

\section{Example 2:}

In this example, the initial guess of $\left(\mu_{1}, \sigma_{0}\right)$ was set to a value far from the true value $(1,1)$. The evolutions of the MCMC sampled $\mu_{1}$ and $\sigma_{0}$ are shown in Figure 3 and 4, respectively, and the recovered result is shown in Table 3. Again, the recovery of $\left(\mu_{1}, \sigma_{0}\right)$ is good. The divergent recovery of $\left(\mu_{1}, \sigma_{0}\right)$ obtained by the Levenberg-Marquardt algorithm is also shown in Table 3. 
Bayesian inference approach to inverse problems

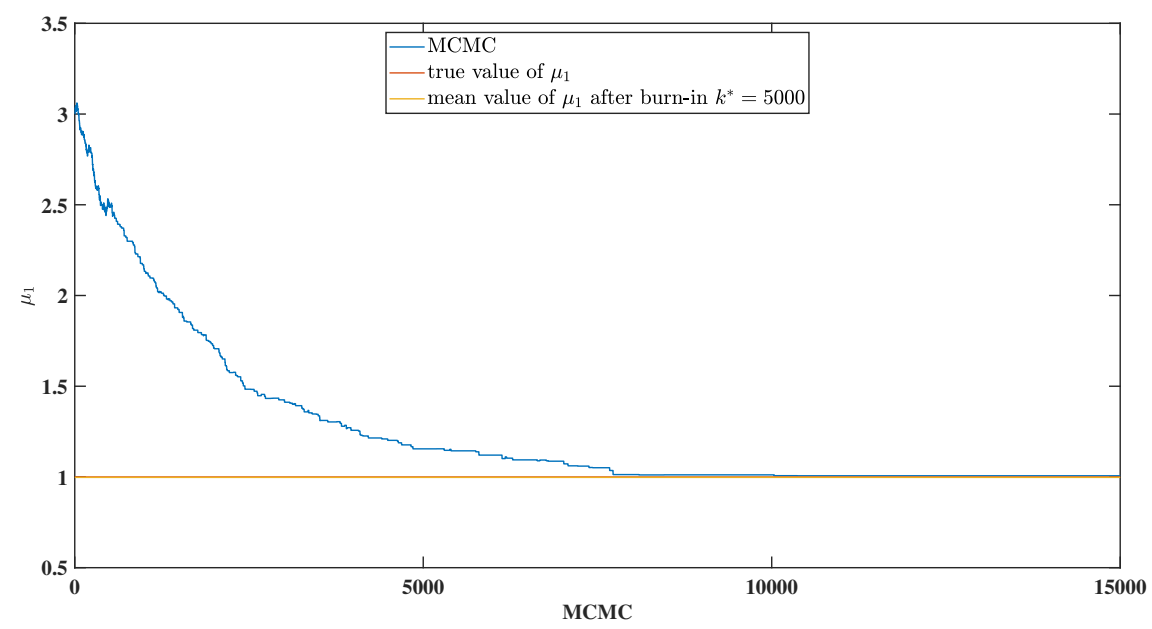

Figure 3: MCMC sampling of the parameter $\mu_{1}$.

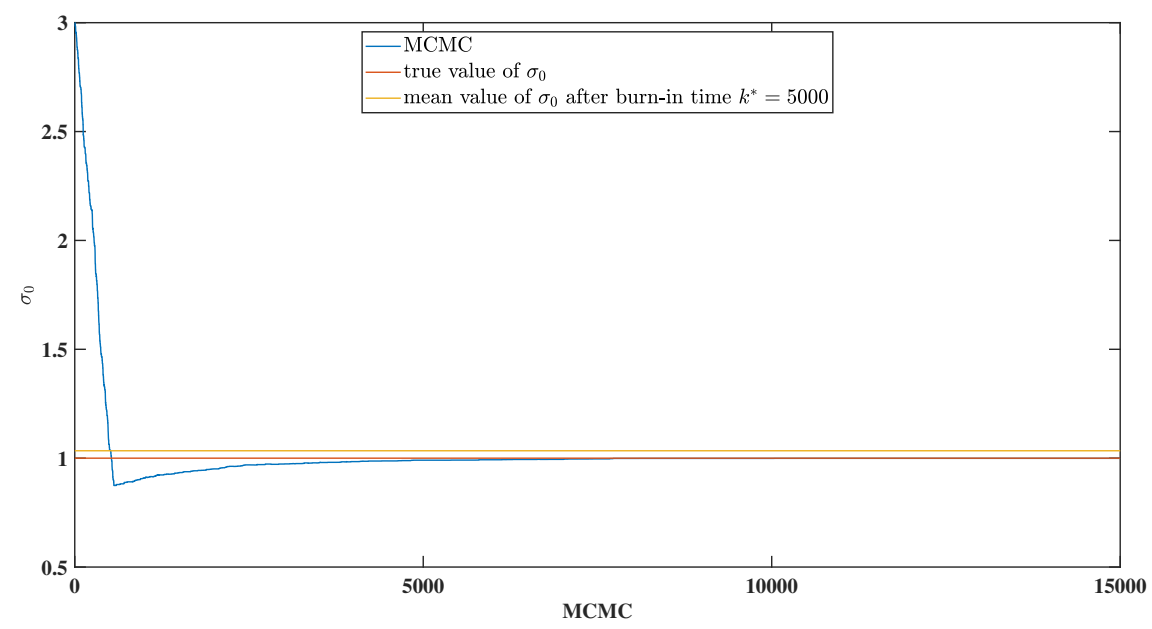

Figure 4: MCMC sampling of the parameter $\sigma_{0}$

Table 3: Recovery results of $\left(\mu_{1}, \sigma_{0}\right)$.

\begin{tabular}{c||c|c}
\hline \hline & $\mu_{1}$ & $\sigma_{0}$ \\
\hline \hline Initial guess & 3 & 3 \\
\hline Mean value after burn-in time $k^{*}=5000$ & 1.0341 & 0.9976 \\
\hline Result of Levenberg-Marquardt algorithm & 4.2334 & 1.0579 \\
\hline True value & 1 & 1 \\
\hline \hline
\end{tabular}


From the MCMC samples in Figures $1-4$ and the recoveries obtained by the Levenberg-Marquardt algorithm (especially in Table 3), we observe that $\mu_{1}$ is more sensitive to noise than $\sigma_{0}$ and hence it is less easily recovered.

\section{Conclusion}

This paper verified the effectively of the Bayesian inference approach to IOP. The posterior distributions of the unknown trend and volatility coefficients were recovered from the measured data by modeling the measurement errors as Gaussian random variables. The posterior state space was explored by the $\mathrm{M}-\mathrm{H}$ method. As confirmed in the numerical results, the Bayesian inference approach simultaneously estimated the unknown trend and volatility coefficients from the measured data.

The method presented here can be extended in several ways. Our immediate future work will statistically apply the Tikhonov regularization method to IOP. As the hierarchical Bayesian method is analogous to the Tikhonov regularization method, it enables the automatic selection of the regularization parameter. Next, we will develop mathematical theories (for instance, the uniqueness, stability, and existence) of IOP and extend our approach to two-dimensional cases. Finally, the model should be tested in a trading strategy using real market data.

\section{Acknowledgments}

The first author would like to acknowledge the supports from JSPS Grantin-Aid for Scientific Research (C) 18K03439. The second author was supported by National Natural Science Foundation of China (No. 11771270). The third author would like to acknowledge the supports from Grant-in-Aid for Scientific Research (15K21766 and 15H05740) of the Japan Society for the Promotion of Science (JSPS).

\section{References}

[1] Black F and Scholes M. 1973 The pricing of options and corporate liabilities Journal of Political Economy. 81, 637-659

[2] Bouchouev I and Isakov V. 1999 Uniqueness, stability and numerical methods for the inverse problem that arises in financial markets Inverse Problems. 15, R95-R116 
Bayesian inference approach to inverse problems

[3] Bouchouev I, Isakov V and Valdivia N. 2002 Recovery of volatility coefficient by linearization Quantitative Finance. Vol2 257-263

[4] Cui T, Fox C, and O?fSullivan MJ. 2011 Bayesian calibration of a large-scale geothermal reservoir model by a new adaptive delayed acceptance Metropolis Hastings algorithm Water Resource Research 47 W10521

[5] Dupire B. 1994 Pricing with a smile Risk. 7 18-20

[6] Doi S and Ota Y. 2018 Application of microlocal analysis to an inverse problem arising from financial markets Inverse Problems. 34 N11

[7] Friedman A. 1983 Partial Differential Equations of Parabolic Type. (Englewood Cliffs, N.J : Prentice-Hall)

[8] Haario H, Laine M, Lehtinen M, Saksman E and Tamminen J. 2004 Markov chain Monte Carlo methods for high dimensional inversion in remote sensing Journal of the Royal Statistical Society: Series B (Statistical Methodology). 66 591-608

[9] Hastings, W. 1970 Monte Carlo sampling methods using Markov chains and their application Biometrika. 57 97-109

[10] Iglesias M A, Lin K and Stuart A M. 2014 Well-posed Bayesian geometric inverse problems arising in subsurface flow Inverse Problems 30, 114001

[11] Kaipio J and Somersalo E. 2005 Statistical and Computational Inverse Problems. (New York : Springer)

[12] Korolev M, Kubo H and Yagola G 2012 Parameter identification problem for a parabolic equation-application to the Black-Scholes option pricing model J. Inverse Ill-posed probl. 20 No.3 327-337

[13] Levenberg, K. 1944 A method for the solution of certain non-linear problems in least squares Quarterly Appl. Math. 2, 164-168

[14] Lishang J and Youshan T. 2001 Identifying the volatility of underlying assets from option prices Inverse Problems. 17 137-155

[15] Marquardt D. 1963. An algorithm for least-squares estimation of nonlinear parameters SIAM J. Appl. Math. 11, 431-441.

[16] Martin J, Wilcox LC, Burstedde C and Ghattas O. 2012 A stochastic Newton MCMC method for large-scale statistical inverse problems with application to seismic inversion SIAM Journal on Scientific Computing 34(3) A1460-A1487 
[17] Metropolis N, Rosenbluth A, Rosenbluth M, Teller A and Teller E. 1953 Equations of state calculations by fast computing machines J. Chem. Phys. 21(6) 1087-1092.

[18] Mitsuhiro M and Ota Y. 2015 Recovery of Foreign Interest Rates from Exchange Binary Options Computer Technology and Application 6 76-88

[19] Ota Y and Kaji S. 2016 Reconstruction of local volatility for the binary option model J. Inverse Ill-posed probl. 24 No.6 727-742

[20] Robert.C and Casella.G 2004 Monte Carlo Statistical Methods (Springer Texts in Statistics)

[21] Wang J and Zabaras N. 2004 A Bayesian inference approach to the inverse heat conduction problem International Journal of Heat and Mass Transfer. 47, Issues 17-18, 3927-3941

[22] Wang J and Zabaras N. 2005 Hierarchical Bayesian models for inverse problems in heat conduction Inverse Problems 21 183-206 